Bull. Austral. Math. Soc.

VoL. 39 (1989) [449-459]

\title{
COMPACTNESS AND CONVEXITY OF CORES OF TARGETS FOR NEUTRAL SYSTEMS
}

\author{
ANThony N. Eke
}

\begin{abstract}
In this paper we prove the convexity and the compactness of the cores of targets for neutral control systems. We make use of a weak compactness argument; but in the crucial part where we establish the boundedness of the cores of the target we make use of the notion of asymptotic direction from Convex Set Theory. Let $E^{n}$ be $n$-dimensional Euclidean space. We prove that the core of the target $H=L+E$ (where $L=\left\{x \in E^{n} \mid M x=0\right\}$, $M$ is a constant $m \times n$ matrix and $E$ is a compact, convex set containing 0 ) of the neutral system

$$
\dot{x}(t)-A \dot{x}(t-h)=B x(t)+C x(t-h)+D u(t)
$$
\end{abstract}

is convex, and is compact if, and only if, the system

$$
\dot{x}(t)-A \dot{x}(t-h)=B^{T} x(t)+C^{T} x(t-h)+M^{T} u(t)
$$

is Euclidean controllable.

\section{INTRODUCTION}

The study of controllability of systems to the core of targets was studied first in the case of linear control systems by Hajek [4].

In this paper, we consider the neutral control system

$$
\begin{cases}\dot{x}(t)-A \dot{x}(t-h) & =B x(t)+C x(t-h)+D u(t) \\ x(t) & =\phi(t), \quad T \in[-h, 0], \quad h>0\end{cases}
$$

where $A, B$ and $C$ are $n \times n$ constant matrices, $D$ is a constant $n \times m$ matrix and $\phi$ is continuous. The control $u$ is an $m$-vector measurable function having values $u(t)$ constrained to lie in a compact, convex, non-empty set $\Omega, \Omega$ being a subset of the Euclidean space $E^{m}$, and $u \in L_{2}([0, t], \Omega)$ for $0<t<\infty$. This $u$ is said to be admissible. The target set $H$ is a closed, convex and non-empty subset of $E^{n}$.

Now suppose $W_{2}^{(1)}$ is the Sobolev space $W_{2}^{(1)}\left([-h, 0], E^{n}\right)$ of functions $\phi:[-h, 0]$ $\rightarrow E^{n}$ which are absolutely continuous with square integrable derivatives. If $x:\left[-h, t_{1}\right] \rightarrow E^{n}$ then, whenever $t \in\left[0, t_{1}\right]$, we write $x_{t}$ as the continuous function on $[-h, 0]$ defined by $x_{t}(s)=x(t+s), s \in[-h, 0]$. Provided $\phi \in W_{2}^{(1)}$ and $u$ is an

Received 21 July, 1988

The author aknowledges with thanks the many useful comments and suggestions of the referee.

Copyright Clearance Centre, Inc. Serial-fee code: 0004-9729/89 \$A2.00+0.00. 
admissible control, there always exists a unique solution for (1.1) such that $x(t)=\phi(t)$ for $t \in[-h, 0]$. This solution is given by the Variation-of-Constants formula

$$
x(t, \phi, u)=x(t, \phi, 0)+\int_{0}^{t} X(t-r) D u(\tau) d \tau,
$$

where the fundamental matrix $X(t)$ satisfies the equation

$$
\begin{gathered}
\dot{x}(t)-A \dot{x}(t-h)=B x(t)+C x(t-h) \\
X(t)= \begin{cases}0, & t<0 \\
1, & t=0\end{cases}
\end{gathered}
$$

and for $t \neq k h, k=0,1,2, \ldots, X(t)$ has a conlinuous first derivative so is of bounded variation on each compact interval $(k h,(k+1) h), k=0,1,2, \ldots$ (see Hale [5, p. 29]). In (1.2) above, we have

$$
\begin{aligned}
& x(t, \phi, 0)=X(t)[\phi(0)-A \phi(-h)]+C \int_{-h}^{0} X(t-\tau-h) \phi(\tau) d \tau \\
&-A \int_{-h}^{0}[d X(t-\tau-h)] \phi(\tau), \quad k \geq h, \quad h>0 .
\end{aligned}
$$

In view of (1.5) above, we can write (1.2) as follows

$$
\begin{aligned}
x(t, \phi, u) & =X(t)[\phi(0)-A 0(-h)]+\int_{0}^{t} X(t-\tau) D u(\tau) d \tau \\
& +C \int_{-h}^{0} X(t-\tau-h) \phi(\tau) d \tau-A \int_{-h}^{0}[d X(t-\tau-h)] \phi(\tau), \quad t>0 .
\end{aligned}
$$

Definition 1.1.. The core of the target set $H$, core $(H)$, is the set of all initial points $\phi(0) \in E^{n}$ for which $\phi \in W_{2}^{(1)}$ such that there exists a measurable control $u:[0, \infty] \rightarrow$ $\Omega$ for which the solution $x(t)=x(t, \phi, u)$ of $(1.1)$ satisfies $x(t) \in H$ for all $t \geq 0$.

Definition 1.2. The system (1.1) is said to be Euclidean controllable if for each $\phi \in$ $W_{2}^{(1)}$ and each $x_{1} \in E^{n}$ there exist a $t_{1} \geqslant 0$ and an admissible control $u$ such that the solution $x(t, \phi, u)=x(t)$, say, of (1.1) satisfies $x_{0}(0, \phi, u)=\phi$ and $x\left(t_{1}, \phi, u\right)=x_{1}$.

Definition 1.3. The system (1.1) is said to be proper on $\left[0, t_{1}\right]$ if and only if $q^{T} X\left(t_{1}-s\right) D=0$ a.e. where $s \in\left[0, t_{1}\right]$, and $q \in E^{n}$ implies $q=0$.

The system (1.1) is controllable on $\left[0, t_{1}\right]$ if and only if it is proper on $\left[0, t_{1}\right]$.

Remark. The above was shown to be true in Chukwu and Silliman [1].

Hence, we have the following lemma

LEMMA 1.1. The system (1.1) is Euclidean controllable on $\left[0, t_{1}\right]$ if and only if $q^{T} X\left(t_{1}-s\right) D=0, q \in E^{n}, s \in\left[0, t_{1}\right]$ implies $q=0$. 


\section{Preliminaries}

We shall give some facts in convex set theory which are crucial to our work. In this section we shall also establish a very important lemma which will be needed in proving the main result of this paper.

Definition 2.1. A point $a \in E^{n}$ is an asymptotic direction of a convex set $S \subseteq E^{n}$ if for $x \in S$ and all $t \geq 0$, we have $x+t a \in S$; that is, the half-ray issuing from $x$ in direction $a$ is entirely contained within $S$.

Proposition 2.1. A non-empty convex set of $E^{n}$ is bounded if and only if 0 is its only asymptotic direction.

Proposition 2.2. Suppose $P \subseteq E^{n}$ is a non-empty convex set of the form $P=$ $L+E$, where $E$ is bounded and contains 0 , and $L$ is a linear subspace of $P$, then $L$ is the largest linear subspace of $P$ and coincides with the set of asymptotic directions of $P$.

Lemma 2.1. If $0 \in H$ and $0 \in \Omega$ then $0 \in \operatorname{core}(H)$ and so core $(H) \neq 0$.

Proof: From (1.6), we have

$$
\begin{aligned}
x(t, \phi, u) & =X(t)[\phi(0)-A 0(-h)]+\int_{0}^{t} X(t-\tau) D u(\tau) d \tau \\
& +C \int_{-h}^{0} X(t-\tau-h) \phi(\tau) d \tau-A \int_{-h}^{0}[d X(t-\tau-h)] \phi(\tau), \quad t \geq 0
\end{aligned}
$$

We choose $\phi(\cdot)=0 \in H, u=0 \in \Omega$ so that we get from (2.1) above

$$
x(t, 0,0)=X(t) 0+\int_{0}^{t} X(t-\tau) D 0 d \tau+0+0=0, \quad t \geq 0
$$

Thus for $0 \in H$ we get $x(t, 0,0)=0 \in H, t \geq 0$. This shows that $\phi(0)=0 \in \operatorname{core}(H)$ and so $\operatorname{core}(H) \neq 0$.

LEMMA 2.2. $a \in E^{n}$ is an asymptotic direction of core $(H)$ if and only if $X(t-s) a$ is an asymptotic direction of $H$.

Proof: Now, for fixed $t, s$ we can write (1.6) as

$$
\begin{aligned}
& x(t-s, \phi, u)=X(t-s)[\phi(0)-A \phi(-h)]+\int_{0}^{t-s} X(t-s-\tau) D u(\tau) d \tau \\
& \quad+C \int_{-h}^{0} X(t-s-\tau-h) \phi(\tau) d \tau-A \int_{-h}^{0}[d X(t-s-\tau-h)] \phi(\tau), \quad t-s \geqslant 0 .
\end{aligned}
$$


We can take an asymptotic direction $a \in \operatorname{core}(H)$ and choose $\phi(0) \in$ core $(H)$ so that for all $\theta \geq 0$ we have $\phi(0)+\theta a \in \operatorname{core}(H)$. We choose an appropriate admissible control $u_{\theta}:[0, \infty) \rightarrow \Omega$ such that the right hand side equals

$$
\begin{aligned}
& X(t-s)[\phi(0)+\theta a-A \phi(-h)]+\int_{0}^{t-s} X(t-s-\tau) D u_{\theta}(\tau) d \tau \\
& \quad+C \int_{-h}^{0} X(t-s-\tau-h) \phi(\tau) d \tau-A \cdot \int_{-h}^{0}[d X(t-s-\tau-h)] \phi(\tau) \in H
\end{aligned}
$$

for $t-s \geqslant 0$.

Dividing throughout by $\theta$ we obtain

$$
\begin{aligned}
& X(t-s)\left[\frac{\phi(0)}{\theta} a-\frac{A}{\theta} \phi(-h)\right]+\frac{1}{\theta} \int_{0}^{t-s} X(t-s-\tau) D u_{0}(\tau) d \tau \\
+ & \frac{C}{\theta} \int_{-h}^{0} X(t-s-\tau-h) \phi(\tau) d \tau-\frac{A}{\theta} \int_{-h}^{0}[d X(t-s-\tau-h)] \phi(\tau) \in \frac{H}{\theta}, \quad t-s \geq 0 .
\end{aligned}
$$

Since $C$ and $A$ are constants we have

$$
\lim _{\theta \rightarrow \infty} \frac{C}{\theta} \int_{-h}^{0} X(t-s-\tau-h) \phi(\tau) d \tau=0
$$

and

$$
\lim _{\theta \rightarrow \infty} \frac{A}{\theta} \int_{-h}^{0}[d X(t-s-\tau-h)] \phi(\tau)=0 .
$$

Also, $\lim _{\theta \rightarrow \infty}(A / \theta) \phi(-h)=0$ and as $\phi(0) \in E^{n}, \lim (\phi(0) / \theta)=0$.

Finally, since the control $u_{\theta}$ is measurable and it is defined on a bounded set, we have

$$
\lim _{\theta \rightarrow \infty} \frac{1}{\theta} \int_{0}^{t-s} X(t-s-\tau) D u_{\theta}(\tau) d \tau=\lim _{\theta \rightarrow \infty} \int_{0}^{t-s} X(t-s-\tau) D \frac{u_{\theta}}{\theta} d \tau=0
$$

Taking limits, we obtain

$$
X(t-s) a=\lim _{\theta \rightarrow \infty} \frac{1}{\theta} b_{\theta} \text { for some } b_{\theta} \in H
$$

We claim that $X(t-s) a$ in (2.2) above is an asymptotic direction of $H$. Indeed, for $c \in H, \lambda \geq 0$, it is sufficient to show that $c+\lambda X(t-s) a \in H$ provided (2.2) above is satisfied. Assuming $\lambda$ is fixed and $\theta \geq \lambda$, we have $\lambda \leq \theta$, that is, $0 \leq \lambda \leq \theta$ and so $0 \leq(\lambda / \theta) \leq 1$ 
Since $H$ is convex, $c \in H, b_{\theta} \in H$, then we have

$$
\left(1-\frac{\lambda}{\theta}\right) C+\frac{\lambda}{\theta} b_{\theta} \in H
$$

In (2.3) above, we take limits as $\theta \rightarrow \infty$ and since $H$ is closed, the limit points of $H$ also belong to $H$.

Therefore $\lim _{\theta \rightarrow \infty}(1-(\lambda / \theta)) c+\lambda \lim _{\theta \rightarrow \infty}(1 / \theta) b_{\theta} \in H$, or $c+\lambda X(t-s) a \in H$, since from (2.2), we have $\lim _{\theta \rightarrow \infty}(1 / \theta) b_{\theta}=X(t-s) a$. This concludes the proof of the claim. Conversely, let $X(t-s) a$ be an asymptotic direction of $H$. For $t-s \geq 0$, we have

$$
H+\theta X(t-s) a \in H, \quad \theta \geq 0
$$

Take $\phi(0) \in$ core $(H)$. Now, choose an admissible control $u_{0}:[0, \infty) \rightarrow \Omega$ such that

$X(t-s)[\phi(0)-A \phi(-h)]+\int_{0}^{t-s} X(-s-\tau) D u_{0}(\tau) d \tau$

$$
+C \int_{-h}^{0} X(t-s-\tau-h) \phi(\tau) d \tau-A \int_{-h}^{0}[d X(t-s-\tau-h) \phi(\tau) \in H, \quad t-s \geq 0 .
$$

If $X(t-s) a$ is an asymptotic direction of $H$, then for all $\theta \geq 0$, in view of definition 2.1 , we have

$$
\begin{gathered}
X(t-s)[\phi(0)-A \phi(-h)]+\int_{0}^{t-s} X(t-s-\tau) D u_{0}(\tau) d \tau+C \int_{-h}^{0} X(t-s-\tau) \phi(\tau) d \tau \\
-A \int_{-h}^{0}[d X(t-s-\tau-h)] \phi(\tau)+\theta X(t-s) a \text { belongs to } H \text { for } t-s \geq 0
\end{gathered}
$$

that is,

$$
\begin{aligned}
X(t-s)[\phi(0)+ & \theta a-A \phi(-h)]+\int_{0}^{t-s} X(t-s-\tau) D \iota_{0}(\tau) d \tau \\
& +C \int_{-h}^{0} X(t-s-\tau-h) \phi(\tau) d \tau-A \int_{-h}^{0}[d X(t-s-\tau-h)] \phi(\tau)
\end{aligned}
$$

belongs to $H$ and from this we infer that $\phi(0)+\theta a \in$ core $(H)$. Now since the same control $u_{0}$ holds this point within $H$, this implies that $a$ is an asymptotic direction of $\operatorname{core}(H)$. 


\section{MaIN RESUlts}

Theorem 3.1. Consider the linear neutral control system (1.1) in which the control $u$ is an m-vector measurable function having values $u(t)$ lying in a compact, convex, non-empty set $\Omega$. Then the core of the target set $H$ ( $H$ being a closed, convex, non-empty subset of $\left.E^{n}\right)$, core $(H)$, is convex.

Proof: Suppose $\phi_{1}(0), \phi_{2}(0) \in$ core $(H)$. Then to two admissible controls, $u_{1}$ and $u_{2}$, there correspond two solutions, $x\left(t, \phi_{1}, u_{1}\right)$ and $x\left(t, \phi_{2}, u_{2}\right)$ such that

$$
\begin{aligned}
& x\left(t, \phi_{i} u_{i}\right)=X(t)\left[\phi_{i}(0)-A \phi_{i}(-h)\right]+\int_{0}^{t} X(t-\tau) u_{i}(\tau) d \tau \\
& +C^{\prime} \int_{-h}^{0} X(t-\tau-h) \phi_{i}(\tau) d \tau-A \int_{-h}^{0}[d X(t-\tau-h)] \phi_{i}(\tau) \in H, \text { for } i=1,2
\end{aligned}
$$

Suppose $\alpha$ is a constant such that $0 \leq \alpha \leq 1, \alpha$ being a constant.

Since the target set $H$ is convex, and since each of $x\left(t, \phi_{i}, u_{i}\right)$, for $i=1,2$, belongs to $H$, then a convex combination of (3.1) belongs to $H$. Thus we have

$$
\alpha x\left(t, \phi_{1}, u_{1}\right)+(1-\alpha) x\left(t, \phi_{i}, u_{i}\right) \in H
$$

that is,

$$
\begin{gathered}
\alpha X(t)\left[\phi_{1}(0)-A \phi_{1}(-h)\right]+\alpha \int_{0}^{t} X(t-\tau) D u_{1}(\tau) d \tau \\
+C \int_{-h}^{0} X(t-\tau-h) \phi_{1}(\tau) d \tau-A \int_{-h}^{0}[d X(t-\tau-h)] \phi_{1}(\tau) \\
+(1-\alpha) X(t)\left[\phi_{2}(0)-A \phi_{2}(-h)\right]+(1-\alpha) \int_{0}^{t} X(t-\tau) D u_{2}(\tau) d \tau \\
+(1-\alpha) C \int_{-h}^{0} X(t-\tau-h) \phi_{2}(\tau) d \tau \\
-(1-\alpha) A \int_{-h}^{0}[d X(t-\tau-h)] \phi_{2}(\tau) \in H
\end{gathered}
$$

Since $\alpha$ is a constant, we can re-arrange (3.2) to obtain

$$
\begin{aligned}
& \text { (3.3) } X(t)\left[\left\{\alpha \phi_{1}+(1-\alpha) \phi_{2}\right\}(0)-A\left\{\alpha \phi_{1}(-h)+(1-\alpha) \phi_{2}(-h)\right\}\right] \\
& +\int_{0}^{t} X(t-\tau) D\left[\alpha u_{1}+(1-\alpha) u_{2}\right](\tau) d \tau+C \int_{-h}^{0} X(t-\tau-h)\left[\alpha \phi_{1}+(1-\alpha) \phi_{2}\right](\tau) d \tau \\
& -A \int_{-h}^{0}\left[d X(t-\tau-h)\left\{\alpha \phi_{1}+(1-\alpha) \phi_{2}\right](\tau) \in H .\right.
\end{aligned}
$$


Since $\Omega$ is a convex set, there is an admissible control $\bar{u}$ such that $\bar{u}(\tau)=\alpha u_{1}(\tau)+$ $(1-\alpha) u_{2}(\tau)$. Also, since $\phi_{i}(\tau) \in E^{n}$ and $E^{n}$ is convex, it follows that there exists $\bar{\phi}$ as follows

$$
\bar{\phi}(-h)=\alpha \phi_{1}(-h)+(1-\alpha) \phi_{2} \quad \text { and } \quad \bar{\phi}(\tau)=\alpha \phi_{1}(\tau)+(1-\alpha) \phi_{2}(\tau) .
$$

When these facts are taken into account in (3.3) above, we see that

$$
\alpha \phi_{1}(0)+(1-\alpha) \phi_{2}(0) \in \operatorname{core}(H) .
$$

This shows that core $(H)$ is convex.

THEOREM 3.2. Consider the neutral control system (1.1). The control functions $u:[0, \infty) \rightarrow \Omega$ are square integrable on finite intervals. The target set $H$ is a closed, convex and non-empty subset of $E^{n}$. Then, the core of the target $H$, core $(H)$, is closed.

Proof: The admissible controls $\mid M$ given by the set

$$
\mid M=\left\{u: u \in L_{2}([0, t], \Omega)\right\}
$$

where $u$ is square integrable, is a closed, convex and bounded subset of $L_{2}\left([0, t], E^{m}\right)$. The space $L_{2}\left([0, t], E^{m}\right)$ is reflexive and so from $[3, p .425]$ we infer that $\mid M$ is weakly compact.

Now, let $\phi_{k}(0)$, for $k=1,2, \ldots$ be a sequence of points belonging to $\operatorname{core}(H)$ with $\phi_{k} \in W_{2}^{(1)}$ the corresponding functions such that

$$
\lim _{k \rightarrow \infty} \phi_{k}=\phi \text { in } W_{2}^{(1)} .
$$

Thus in $E^{n} \lim _{k \rightarrow \infty} \phi_{k}(0)=\phi(0)$ and $\lim _{k \rightarrow \infty} \phi_{k}(-h)=\phi(-h)$. Let $u_{k}$, for $k=1,2, \ldots$ be the corresponding admissible controls such that for $k=1,2, \ldots$ we have

$$
\begin{aligned}
& x\left(t, \phi_{k}, u_{k}\right)=X(t)\left[\phi_{k}(0)-A \phi_{k}(-h)\right]+\int_{0}^{t} X(t-\tau) D u_{k}(\tau) d \tau \\
& \quad+C \int_{-h}^{0} X(t-\tau-h) \phi_{k}(\tau) d \tau-A \int_{-h}^{0}[d X(t-\tau-h)] \phi_{k}(\tau) \in H, \quad t \geqslant 0
\end{aligned}
$$

Since $\mid M$ is weakly compact, there exists a subsequence $u_{k j}$ of $u_{k}$, with $j=1,2, \ldots$ which converges weakly to a control function $\bar{u}_{0} \in \mid M$ on $\left[0, t_{1}\right]$. .

In other words,

$$
\lim _{j \rightarrow \infty} \int_{0}^{t} X(t-\tau) D u_{k j}(\tau) d \tau=\int_{0}^{t} X(t-\tau) D \bar{u}_{0}(\tau) d \tau
$$


Suppose now that $\left\{\phi_{k j}\right.$, for $\left.j=1,2, \ldots\right\}$, are the subsequences of $\left\{\phi_{k}\right.$, for $k=$ $1,2, \ldots\}$ corresponding to $\left\{u_{k j}\right.$, for $\left.j=1,2, \ldots\right\}$. Then we have

$$
\begin{aligned}
& x\left(t, \phi_{k j}, u_{k j}\right)=X(t)\left[\phi_{k j}(0)-A \phi_{k j}(-h)\right]+\int_{0}^{t} X(t-\tau) D u_{k j}(\tau) d \tau \\
& +C \int_{-h}^{0} X(t-\tau-h) \phi_{k j}(\tau) d \tau-A \int_{-h}^{0}[d X(t-\tau-h)] \phi_{k j}(\tau) \in H, \quad t \geqslant 0
\end{aligned}
$$

Since $H$ is closed, if we take the limits of both sides of (3.7) these limits belong to $H$; that is

$$
\begin{aligned}
\lim _{j \rightarrow \infty} x\left(t, \phi_{k j}, u_{k j}\right) & =\lim _{j \rightarrow \infty} X(t)\left[\phi_{k j}(0)-A \phi_{k j}(-h)\right]+\lim _{j \rightarrow \infty} \int_{0}^{t} X(t-\tau) D u_{k j}(\tau) d \tau \\
& +\lim _{j \rightarrow \infty} C \int_{-h}^{0} X(t-\tau) \phi_{k j}(\tau) d \tau-\lim _{j \rightarrow \infty} A \int_{-h}^{0}[d X(t-\tau-h)] \phi_{k j}(\tau) \in H .
\end{aligned}
$$

Thus from (3.4), (3.6) and (3.8) we have

$$
\begin{aligned}
\lim _{j \rightarrow \infty} x\left(t, \phi_{k j}, u_{k j}\right)= & X(t)\{\phi(0)-A \phi(-h)]+\int_{0}^{t} X(t-\tau) D \bar{u}_{0}(\tau) d \tau \\
& +C \int_{-h}^{0} X(t-\tau-h) \phi(\tau) d \tau-A \int_{-h}^{0}[d X(t-\tau-h)] \phi(\tau) \in H
\end{aligned}
$$

which implies that $\phi(0) \in \operatorname{core}(H)$ and so core $(H)$ is closed.

ThEOREM 3.3. Let us consider the neutral control system

$$
\begin{cases}\dot{x}(t)-A \dot{x}(t-h) & =B x(t)+C x(t-h)+D u(t) \\ x(t) & =\phi(t), t \in[-h, 0], \text { and } h>0\end{cases}
$$

Suppose the target set $H$ is of the form $H=L+E$, with $L=\left\{x \in E^{n}: M x=0\right\}$ a linear subspace of $H$, and $E$ a compact, convex set containing 0 of the control system (1.1) and $M$ is an $m \times n$ constant matrix. Let $0 \in \Omega$ and also $0 \in H$. Under these conditions, core $(H)$ is compact if and only if the control system

$$
\dot{x}(t)-A \dot{x}(t-h)=B^{T} x(t) C^{T} x(t-h)+M^{T} u(t)
$$

is Euclidean controllable.

Proof: Suppose $\left\{\phi_{n}(0) \mid n=1,2, \ldots\right\}$ is the set of asymptotic directions of $\operatorname{core}(H)$. Then Lemma 2.2 implies that $\left\{X(t-s) \phi_{n} \mid n=1,2, \ldots\right\}$ is the set of 
asymptotic directions of $H$. From Proposition 2.2, which says that $L$ coincides with the set of asymptotic directions of $H$, we conlude that $L=\left\{X(t-s) \phi_{n}(0) \mid n=1,2, \ldots\right\}$.

The hypothesis on $H$ in the above theorem implies that

$$
M X(t-s) \phi_{n}(0)=0
$$

Taking the transposes, we have

$$
\phi_{n}^{T}(0) X^{T}(t-s) M^{T}=0, \text { for all } n, \quad t-s \geqslant 0 .
$$

Let us suppose now that the system

$$
\dot{x}(t)-A \dot{x}(t-h)=B^{T} x(t)+C^{T} x(t-h)+M^{T} u(t)
$$

is Euclidean controllable on $\left[0, t_{1}\right]$ for each $t_{1}>0$. Then by Lemma 1.1 this means that $\phi_{n}^{T}(0) X^{T}(t-s) M^{T}=0, \phi_{n}(0) \in E^{n}$ implies $\phi_{n}(0)=0, \forall t-s \geqslant 0$, for each $n$. Hence by hypothesis, this shows that 0 is the only asymptotic direction of core $(H)$. Lemma 2.1 gives that core $(H)$ is non-empty. Also Theorem 3.1 shows that core $(H)$ is convex. Thus, core $(H)$ is a non-empty convex subset of $E^{n}$ with 0 as its only asymptotic direction; then Proposition 2.1 implies that core $(H)$ is bounded. But Theorem 3.2 shows that core $(H)$ is also closed. Thus core $(H)$ is compact.

Conversely, assume that core $(H)$ is compact. This implies that core $(H)$ is bounded. So Proposition 2.1 gives that 0 is the sole asymptotic direction. Refferring now to (3.9) above, we have that $\phi_{n}^{T}(0) X^{T}(t-s) M^{T}=0$ implies $\phi_{n}(0)=0$ for all $t-s \geqslant 0$, and for all $n$. Hence Lemma 1.1 implies by this that the control system

$$
\dot{x}(t)-A \dot{x}(t-h) B^{T} x(t)+C^{T} x(t-h)+M^{T} u(t)
$$

is Euclidean controllable on $\left[0, t_{1}\right]$, for $t_{1} \geqslant 0$. We have thus proved our main result.

\section{EXAMPLE}

Consider in $E^{2}$, the $x-y$ plane, say, the target set $H$ defined by

$$
H=\left\{x=\left(\begin{array}{l}
x_{1} \\
x_{2}
\end{array}\right) \mid x_{1}=0, x_{2} \neq 0\right\}
$$

where $x \in E^{2}$. Then systems of vectors of the form $\left(\begin{array}{l}0 \\ \eta\end{array}\right)$ for all finite non-zero entries $\eta \in E^{\mathbf{1}}$ belong to core $(H)$. Thus any neutral control system in $E^{2}$ of the form (1.1) with initial function $\phi_{0} \in W_{2}^{(1)}\left([-1,0], E^{2}\right)$ such that

$$
\phi_{0}(t)=\left\{\left(\begin{array}{l}
\phi_{1}(t) \\
\phi_{2}(t)
\end{array}\right) \mid \phi_{1}(t)=0, \phi_{2}(t) \neq 0 \text { for all } t \geq 0\right\}
$$


implies that $\phi_{0}(t) \in$ core $(H)$.

Following Theorem 3.1, we infer that this core $(H)$ is convex and it is definitely bounded. In ( 4.1$)$ above we define $M=\left(\begin{array}{ll}1 & 0\end{array}\right)$, a $1 \times 2$ constant matrix.

Now, consider the neutral system in $E^{2}$ given as

$$
\dot{x}(t)-A \dot{x}(t-1)=B x(t)+C x(t-1)+D u(t)
$$

where

$$
A=\left(\begin{array}{ll}
1 & 0 \\
0 & 1
\end{array}\right), \quad B=\left(\begin{array}{cc}
0 & 0 \\
1 & -2
\end{array}\right), \quad C=\left(\begin{array}{cc}
-1 & -1 \\
0 & -1
\end{array}\right), \quad D=\left(\begin{array}{l}
1 \\
0
\end{array}\right)
$$

which satisifes the initial condition (4.2) and has the target set (4.1) above.

Following Hale [5, p.144] we need to find the fundamental matrix $X(t-s)$ of (4.3). With the data for the system (4.3) we obtain, after lengthy but straightforward calculations as in Driver [2],

$$
X(t-s)=e^{2 \tau}\left(\begin{array}{cc}
1+\tau & -\tau \\
\tau & 1-\tau
\end{array}\right)
$$

for some $\tau=s-T \geq 0$, where $T \geq 0$, for which the $u$ in (4.3) is admissible. Choosing any $\xi=\left(\begin{array}{l}\xi_{1} \\ \xi_{2}\end{array}\right) \in E^{2}$ we see that

$$
e^{-2 \tau}\left(\xi_{1}, \xi_{2}\right)\left(\begin{array}{cc}
1+\tau & \tau \\
-\tau & 1-\tau
\end{array}\right)\left(\begin{array}{l}
1 \\
0
\end{array}\right)=e^{-2 \tau}\left(\xi_{1}+\tau \xi_{1}-\tau \xi_{2}\right)=0
$$

is true if and only if $\xi_{1}=0$ and $\xi_{2}=0$, which implies

$$
\xi=\left(\begin{array}{l}
\xi_{1} \\
\xi_{2}
\end{array}\right)=0
$$

That is,

$$
\xi^{T} X^{T}(t-s) M^{T}=0 \text { implies } \xi=0
$$

which, in turn, inplies by Lemma 1.1 that the system

$$
\dot{x}(t)-A \dot{x}(t-1)=B^{T} x(t)+C^{T} x(t-1)+M^{T} u(t)
$$

is Euclidean coutrollable. 


\section{REFERENCES}

[1] E.N. Chukwu and S.D. Silliman, 'Constrained controllability to a closed target set', J: Optim. Theory Appl. 21 (1978), 369-383.

[2] H.D. Driver, Ordinary and Delay Differential Equations, Appl. Math. Sci., vol 20 (Springer-Verlag, Berliu, Heidelberg, New York, 1976).

[3] M. Dunford and J.T. Schwarts, Linear Operators, Part 1 (John Wiley and Sons, Interscience, New York, 1958).

[4] O. Hajek, 'Cores of targets in linear control systems', Math. Systems Theory 8 (1974), $203-206$.

[5] Jack Hale, Theory of Functional Differential Equations, Appl. Math. Sci., vol 3 (Springer-Verlag, Berlin, Heidleberg, New York, 1977).

Department of Mathematics,

University of Nigeria,

Nsukka,

Nigeria. 